\title{
ORBITS OF FAMILIES OF VECTOR FIELDS AND INTEGRABILITY OF SYSTEMS WITH SINGULARITIES
}

\author{
BY HECTOR J. SUSSMANN
}

Communicated by A. P. Calderón, June 9, 1972

Let $X$ be a $C^{\infty}$ vector field on the $C^{\infty}$ manifold $M$. We use $t \rightarrow X_{t}(m)$ to denote the integral curve of $X$ which passes through $m$ when $t=0$. Let $D$ be a set of $C^{\infty}$ vector fields on $M$. Two points $m$ and $m^{\prime}$ of $M$ are said to be $D$-connected if there exist elements $X^{1}, \ldots, X^{k}$ of $D$ and real numbers $t_{1}, \ldots, t_{k}$ such that

$$
m^{\prime}=X_{t_{1}}^{1}\left(X_{t_{2}}^{2}\left(\cdots X_{t_{k}}^{k}(m) \cdots\right)\right) \text {. }
$$

This defines an equivalence relation on $M$. The equivalence classes are called the orbits of $D$.

Let $S$ be an orbit of $D$. For each $m \in S$ and each finite sequence $\xi=\left(X^{1}, \ldots, X^{k}\right)$ of elements of $D$, let $F_{\xi, m}$ denote the map

$$
\left(t_{1}, \ldots, t_{k}\right) \rightarrow X_{t_{1}}^{1}\left(X_{t_{2}}^{2}\left(\cdots X_{t_{k}}^{k}(m) \cdots\right)\right) \text {. }
$$

It is clear that $F_{\xi, m}$ is a $C^{\infty}$ mapping from an open subset $U$ of $R^{k}$ into $M$. Moreover the range of $F_{\xi, m}$ is a subset of $S$. We topologize $S$ by the strongest topology for which all the maps $F_{\xi, m}$ are continuous.

THEOREM $1 . S$ is a connected $C^{\infty}$ submanifold of $M$.

A distribution on $M$ is a mapping $H$ which assigns to every $m \in M$ a linear subspace $H(m)$ of the tangent space of $M$ at $m$. It is not required that the dimension of $H(m)$ be constant. A vector field $X$ defined in an open subset $U$ of $M$ belongs to the distribution $H$ if $X(m) \in H(m)$ for every $m \in U$. We say that $H$ is a $C^{\infty}$ distribution if, for every $m \in M$ and every $v \in H(m)$, there exists a $C^{\infty}$ vector field $X$ such that $X$ belongs to $H$ and $X(m)=v$. If $D$ is a set of vector fields and $H$ is a distribution, we say that $H$ is $D$-invariant if, whenever $m \in M, X \in D$, and $t$ is a real number such that $X_{t}(m)$ is defined, it follows that the differential of $X_{t}$ maps $H(m)$ into $H\left(X_{t}(m)\right)$. Given a set $D$ of $C^{\infty}$ vector fields on $M$, there exists a smallest distribution $H$ which is $D$-invariant and is such that every element of $D$ belongs to $H$. Let this distribution be denoted by $P_{D}$. Then $P_{D}$ is a $C^{\infty}$ distribution.

Integral manifolds and maximal integral manifolds of a distribution

AMS (MOS) subject classifications (1970). Primary 49E15, 58A30.

Key words and phrases. Orbits, leafs, vector distributions with singularities, complete integrability, attainable sets. 
are defined in the usual way. In the statement of the following theorem, the orbits of $D$ are given the topology that was defined in the remarks preceding Theorem 1, and the differentiable structure whose existence is asserted in Theorem 1. This structure is clearly unique.

THEOREM 2. The orbits of $D$ are maximal integral manifolds of $P_{D}$.

The preceding result shows that the distribution $P_{D}$ is integrable, in the sense that through every point of $M$ there passes a maximal integral manifold of $P_{D}$. It turns out that the converse is also true: Every integrable distribution is of the form $P_{D}$ for some $D$.

THEOREM 3. Let $H$ be a $C^{\infty}$ distribution and let $D$ be a set of $C^{\infty}$ vector fields such that, for every $m \in M, H(m)$ is the linear hull of the vectors $X(m)$, $X \in D$. Then the following conditions are equivalent:

(a) Through every point of $M$ there passes an integral manifold of $H$.

(b) Through every point of $M$ there passes a maximal integral manifold of $H$.

(c) $H=P_{D}$.

(d) For every $m \in M$ there exist elements $X^{1}, \ldots, X^{k}$ of $D$ such that $H(m)$ is the linear hull of $X^{1}(m), \ldots, X^{k}(m)$ and that the following holds:

For every $X \in D$ there exist $\varepsilon>0$ and $C^{\infty}$ functions

$$
f_{j}^{i}:(-\varepsilon, \varepsilon) \rightarrow R \quad(1 \leqq i, j \leqq k)
$$

such that, for $-\varepsilon<t<\varepsilon$,

$$
\left[X, X^{i}\right]\left(X_{t}(m)\right)=\sum_{i=1}^{k} f_{j}^{i}(t) X^{j}\left(X_{t}(m)\right) .
$$

Proofs of our three theorems will appear elsewhere. We now discuss the connection of our results with those of Chow [1], Hermann [2], Lobry [3], Nagano [5] and Matsuda [4]. Let $D^{*}$ be the smallest set of vector fields which contains all the elements of $D$ and which is closed under Lie brackets. Let $I_{D}(m)$ denote the linear hull of the vectors $X(m), X \in D^{*}$. Chow proved that the orbits of $D$ are precisely the connected components of $M$, provided $I_{D}(m)$ has maximal dimension for each $m$. If the assumption of Chow's theorem is satisfied, it is clear that $I_{D}(m)=P_{D}(m)=$ tangent space of $M$ at $m$. Therefore Chow's result is a particular case of Theorem 2. Hermann and Lobry studied (under the name of "leafs") the orbits of $D$ under the assumption that the distribution $I_{D}$ is integrable. By applying Chow's theorem to the integral manifolds of $I_{D}$, they concluded that the orbits of $D$ are the maximal integral manifolds of $I_{D}$. Theorem 1 shows that the orbits of $D$ are always smooth submanifolds of $M$, and that the integrability of $I_{D}$ is not needed. Theorem 2 shows that the orbits are the maximal integral manifolds of an integrable distribution $P_{D}$. In general, 
$P_{D}$ will not coincide with $I_{D}$ and, therefore, $I_{D}$ is not the "correct" distribution to look at.

The integrability of smooth distributions $H$ which are involutive (i.e., whenever $X$ and $Y$ belong to $H$ then $[X, Y]$ belongs to $H$ ) is a classical result if the dimension of $H(m)$ is constant. In general (i.e., when $H$ has "singularities") the condition that $H$ be involutive is necessary but not sufficient. Sufficient conditions were given by Hermann [2] ( $H$ is "locally finitely generated"), Lobry [3] ( $H$ is "locally of finite type") and Matsuda [4] ( $H$ satisfies a "convergence condition"). It is easy to see that Lobry's condition (which is weaker than Hermann's) implies condition (d) of Theorem 3. Also, Matsuda's condition implies (c). Therefore, these results are all contained in Theorem 3.

Nagano [5] proved the result (stated by Hermann in [2]) that every analytic involutive distribution on a real analytic manifold $M$ is integrable. This also follows from Theorem 3, because in the analytic case condition (d) is always satisfied (cf. Lobry [3]).

\section{REFERENCES}

1. W. L. Chow, Uber Systeme von linearen partiellen Differentialgleichungen erster Ordnung, Math. Ann. 117 (1939), 98-105.

2. R. Hermann, On the accessibility problem in control theory, Internat. Sympos. Nonlinear Differential Equations and Nonlinear Mechanics, Academic Press, New York, 1963 , pp. 325-332. MR 26 \#6891.

3. Claude Lobry, Contrôlabilité des systèmes non lineaires, SIAM J. Control 8 (1970), 573-605.

4. M. Matsuda, An integration theorem for completely integrable systems with singularities, Osaka J. Math. 5 (1968), 279-283. MR 39 \#4876.

5. T. Nagano, Linear differential systems with singularities and an application to transitive Lie algebras, J. Math. Soc. Japan 18 (1966), 398-404. MR 33 \#8005.

Department of Mathematics, University of Chicago, Chicago, Illinois 60637

Department of Mathematics, Rutgers University, New Brunswick, New Jersey 08903 (Current address) 